\title{
Les nouveaux enjeux territoriaux de la réforme de la carte militaire
}

The new territorial stakes of military map reform

\section{Florian Rapin}

\section{(2) OpenEdition}

\section{Journals}

Édition électronique

URL : http://journals.openedition.org/rge/3257

DOI : $10.4000 /$ rge.3257

ISSN : 2108-6478

\section{Éditeur}

Association des géographes de l'Est

Édition imprimée

Date de publication : 30 mai 2011

ISSN : 0035-3213

\section{Référence électronique}

Florian Rapin, « Les nouveaux enjeux territoriaux de la réforme de la carte militaire », Revue

Géographique de l'Est [En ligne], vol. 51 / 1-2 | 2011, mis en ligne le 19 décembre 2011, consulté le 08 septembre 2020. URL : http://journals.openedition.org/rge/3257 ; DOI : https://doi.org/10.4000/rge. 3257

Ce document a été généré automatiquement le 8 septembre 2020

Tous droits réservés 


\section{Les nouveaux enjeux territoriaux de la réforme de la carte militaire}

The new territorial stakes of military map reform

Florian Rapin

\section{Introduction}

1 Avec l'élaboration en 2008 d'une nouvelle organisation territoriale les armées connaissent un bouleversement de leurs structures. Cette réforme s'inscrit dans le cadre de la révision générale des politiques publiques (RGPP) à la suite de la réalisation d'un Livre Blanc sur la Défense. La rationalisation des implantations militaires remet en cause le concours des armées à l'aménagement du territoire et les fermetures de site sont un traumatisme pour les villes qui dépendent largement des activités de Défense. Nombreux sont les acteurs qui ont pesé sur l'essor de la réforme, aujourd'hui le temps est à l'action sur les reconversions à mener.

\section{I - Une réforme inscrite dans la Révision Générale des Politiques Publiques.}

\section{A - Une réforme en plusieurs étapes.}

2 En France, la Défense est définie comme globale et complexe et s'articule autour des concepts stratégiques définis dans le Livre Blanc. La nouvelle version de 2008 reprend les piliers de 1993 (prévention, protection, projection et dissuasion ${ }^{1}$ ) et ajoute le renseignement (anticipation et connaissance).

3 Si le Livre Blanc définit le contour des missions, la révision générale des politiques publiques (RGPP) assigne les objectifs quantitatifs au Ministère de la Défense. L'ensemble des dispositions conduit à une réduction du format des armées de 54000 hommes. 
4 En conséquence, l'organisation territoriale de la Défense doit être reconfigurée pour permettre la conduite des missions avec moins de personnels. La rationalisation des moyens humains, financiers et matériels ne doit pas entraver la conduite des missions, le choix est fait de regrouper les unités militaires au sein de Bases de Défense. Il ne s'agit pas ici de la première restructuration que connaisse ce Ministère. L'ouvrage éponyme ${ }^{2} \mathrm{du}$ Général Claude Carré retrace l'évolution historique de la Défense en France, notamment dans son organisation territoriale. Ce qui permet de distinguer la réforme actuelle c'est qu'il s'agit d'une réorganisation en temps de paix. En effet, les réorganisations majeures ont été le fait de modifications substantielles du territoire, à la fois lors d'expansions que lors de contractions à l'image de celle suivant la fin de la " guerre » d'Algérie.

Outre cette réforme en temps de paix, il y a une innovation structurelle avec l'apparition de la Base de Défense (BdD). Dans ce nouveau schéma d'organisation la fonction de soutien est clairement distinguée de la mise en condition opérationnelle. Un commandement du groupement de soutien (GSBdD) est créé et rattaché au commandant de la Base de Défense (ComBdD). Après une phase d'expérimentation en 2010, toutes les Bases de Défense sont entrées en fonction au 1er janvier 2011.

6 La carte présentée en août 2008 mentionnait plus de 70 bases, une concentration supplémentaire a permis de réduire leur nombre à 60 (arrêté du 29 novembre 2010) dont 51 sont situées en métropole. Dans l'aire d'étude considérée on retrouve 7 bases de défense : Brest, Rennes, Coëtquidan, Cherbourg, Angers, Saint-Maixent, Rochefort et Angoulême.

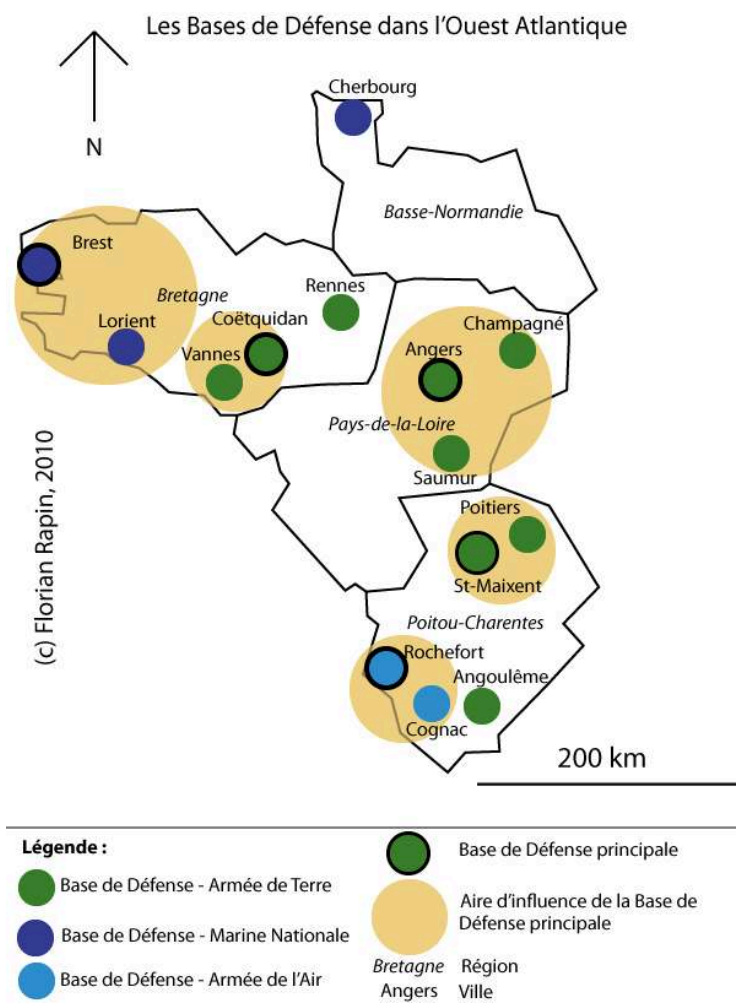

Figure 1 : La carte des Bases de Défense dans I'Ouest Atlantique

(d'après les publications successives du Ministère de la Défense de 2008 à 2010)

7 Cette carte permet de faire état de la concentration supplémentaire réalisée entre la première présentation en 2008 et la mise en œuvre en 2010. Ainsi, certaines bases ont 
été rassemblées mais toujours dans une cohérence de branche sur cet échantillon. L'objectif était d'accroître le volume des personnels soutenus dans une base. D'après le référé en date du 7 mars $2011^{3} \mathrm{du}$ président de la Cour des Comptes, 14 bases soutiennent moins de 3000 personnes. Le contrôle réalisé a permis de mettre en évidence certaines incohérences géographiques dans l'organisation administrative citées dans ce même référé : « les bases de défense de Lille et de Creil sont, par exemple, rattachées à l'état-major de soutien de la défense de Metz et à la plateforme achatsfinances de Saint Germain-en-Laye ».

\section{B - Les différentes comparaisons possibles de cette réforme.}

8 L'étude de cette réforme en cours permet d'envisager plusieurs comparaisons, en premier lieu vis-à-vis d'autres pays mais aussi par rapport à d'autres ministères concernés eux aussi par la RGPP. Ainsi, la constitution de Bases de Défense rapproche l'armée française des modèles américains de Superbase. L'option vers ce type d'organisation avec un nombre restreint de sites correspond à une volonté de disposer de forces de projection dans un contexte de conflits extérieurs plutôt que pour défendre le territoire. Toutefois, l'amélioration des technologies de communication permet d'organiser un maillage du territoire pour répondre en cas de menace. Cette évolution des technologies est à prendre en compte dans les perspectives offertes pour organiser la défense avec moins d'unités.

9 Plusieurs comparaisons doivent s'établir avec des réformes menées dans d'autres ministères à l'image de la Justice qui a connu, avec la RGPP, un mouvement similaire pour rationnaliser ses implantations qu'il s'agisse des tribunaux, avec la réforme de la carte judiciaire, ou bien des prisons avec le plan de rénovation. Ces ministères ont en commun de disposer d'un patrimoine dont la qualité architecturale et la localisation concentrent les intérêts. Par contre, il convient de signaler une différence notable dans le domaine de la communication. En effet, le Ministère de la Défense communique largement sur cette réforme au travers d'un site dédiét, par contre, du côté du Ministère de la Justice, les informations sont plus rares et dispersées alors que le processus de réforme est achevé.

Par ailleurs, l'évolution de la Poste, avec ses effets d'entrainement locaux peut aussi constituer une comparaison intéressante puisqu'il s'agit dans ce cas de l'évolution d'un établissement public industriel et commercial et non d'une administration et que sa valeur symbolique est forte au regard des très nombreuses implantations.

\section{II - Les impacts du nouveau dispositif territorial.}

\section{A - Une dimension économique prégnante.}

11 Cette réforme majeure doit permettre de réaliser des économies importantes pour le budget de la défense. Une part non négligeable, plus d'un milliard d'euros sur les six milliards estimés au total doivent provenir des cessions immobilières. En conséquence, de nombreuses emprises (casernements et terrains d'entraînement) seront libérées en 2009 et 2014 pour une surface totale de 6355 hectares 5 . L'Etat peut souhaiter la conservation de certaines emprises au profit d'autres ministères. C'est le cas de la reconversion de casernements en logements étudiants comme à Arras ou Montpellier. 
La cession peut être consentie aux communes ou à des acteurs privés. Les emprises peuvent aussi être cédées aux communes ou à défaut aux acteurs privés.

Des opérations de reconversion ont déjà été menées par le passé à l'occasion de dissolutions d'unités. La transformation des casernes dans les villes est une opération délicate puisqu'elle est visible des habitants. Toutes les casernes ne furent pas détruites, leur destination finale a pu s'orienter vers l'accueil d'autres administrations ou d'établissements d'enseignement. Ce qui diffère ici c'est l'ampleur des espaces concernés alors que nous sommes en temps de paix.

certains de des territoires, la Défense constitue l'activité première. Pour compenser la perte d'activité, des mesures d'accompagnement ont été mises en place. Les territoires particulièrement affectés peuvent bénéficier d'un contrat de redynamisation de site de Défense. Ces contrats sont mis en place à l'échelle communale ou intercommunale et offrent donc des réponses très ciblées pour compenser les pertes liées au départ d'unités. Des plans locaux de revitalisation sont aussi lancés à l'échelle départementale ou régionale (comme en Pays de la Loire) dans les situations moins graves.

14 Les villes qui accueillent de nouvelles unités peuvent être qualifiées de gagnantes par opposition à celles qui voient les militaires partir. Toutefois, cette vision binaire mérite d'être affinée. En effet, le renforcement lié à la constitution des bases de défense peut créer une tension sur le foncier pour les communes concernées puisqu'il faut pouvoir loger les militaires et civils qui affluent et intégrer la construction de nouveaux bâtiments ou casernes. Il convient aussi d'envisager les bénéfices réels liés à l'extension de l'activité militaire sur le territoire. En effet, si la trop grande dépendance de l'économie locale à l'emploi public est une faiblesse, il faut s'assurer que le surplus d'activités ne se fasse pas au détriment de l'implantation d'industries ou d'entreprises du secteur privé. Par ailleurs, les collectivités concernées par l'accueil d'unités supplémentaires peuvent espérer une amélioration du niveau des services publics dont elles disposent.

Toutefois, l'enjeu essentiel concerne la capacité des villes à réagir à la disparition des militaires. Nantes se situe dans ce cas puisqu'à terme, en dehors des gendarmes, il n'y aura plus de militaires dans la ville. Au regard de la situation actuelle, le départ des militaires de Nantes ne constitue pas un bouleversement dramatique de l'économie locale. Néanmoins, l'objectif des représentants de la ville et de la métropole est de lancer une dynamique pour de réaliser de nouveaux projets. A ce titre, Nantes fait partie des villes qui bénéficient d'un plan local de revitalisation. Ceci doit permettre de dépasser la perte directe liée au départ des militaires et de développer de nouvelles activités, en particulier, dans des espaces stratégiques à proximité du centre.

Pour réaliser le PLR, le Ministère de la Défense passe une commande auprès d'un cabinet d'audit, en l'occurrence SCET/CDID (filiale de la Caisse des Dépôts) dans le cas nantais. Un diagnostic territorial est réalisé de manière à présenter les atouts et faiblesses de l'espace concerné. L'évaluation doit aboutir à des propositions de la part du cabinet pour l'implantation de nouvelles activités, privées pour l'essentiel avec des estimations d'emplois.

L'impact économique lié à l'évolution démographique du fait de la réforme est certain en positif comme en négatif. Les effets d'entraînements seront visibles à des échelles très locales avec le développement des communes périphériques accueillant les 
personnels affectés. On peut imaginer le redéploiement de services publics, comme l'amélioration de la desserte TGV à Saumur avec l'arrivée des stagiaires de l'Ecole d'Etat-Major qui rentrent dans leurs familles le week-end. A l'inverse, les effets négatifs seront ressentis si la reconversion échoue. Dans la mesure où les activités sont multiples, les PLR et CRSD doivent permettre d'accueillir de nouvelles entreprises. Pour le cas des villes dans lesquels la présence d'unités jouait un rôle prépondérant, ce qui se traduit par la mise en place d'un CRSD, l'enjeu est crucial, d'autant qu'il peut se répercuter dans le bassin de vie et entraîner la désertification dans les communes périphériques avec des impacts négatifs comme la fermeture de classes et de service comme La Poste qui se situe aussi dans un contexte de reforme.

Toutefois, l'impact fiscal de cette reforme pour les collectivités n'est en aucun cas abordé. Cette absence est sans doute liée aux difficultés d'évaluation du produit fiscal qui dépend en grande partie de la destination future du site militaire. En effet, les sites militaires sont en partie exonérés d'impôts locaux (taxe d'habitation, sur le foncier bâti et non-bâti). L'installation d'une nouvelle activité modifie le produit fiscal des parcelles concernées. Ainsi, l'arrivée d'une entreprise permet de percevoir les taxes précitées ainsi que la nouvelle contribution économique territoriale qui remplace la taxe professionnelle. Pour les particuliers, le choix de la «verticalisation" permet d'accroître les recettes d'une parcelle. Toutefois, ce surplus fiscal est à mettre en balance avec la demande marginale en services et équipements publics des entreprises ou particuliers nouvellement installés. Cette dimension complique encore l'évaluation qui peut être faite de l'enjeu fiscal de la réforme.

19 Au sujet du monde économique, évoqué précédemment, l'industrie de défense semble peu impactée par la réforme. De fait, la nature et le coût des équipements se traduisent par une procédure centralisée pour les marchés publics. C'est la loi de programmation militaire par l'identification du nouveau format des armées et des budgets d'équipement qui permet d'envisager les impacts sur ce pan de l'industrie. La réforme a été voulue pour rationaliser les chapitres $d u$ fonctionnement afin de privilégier l'investissement pour les armées. Ainsi, une partie des économies réalisée sera réaffectée au budget pour financer l'acquisition et la modernisation de matériels. L'effort en matière de Défense a vocation à rester stable le budget global regroupant fonctionnement et investissement devrait rester constant. Une évaluation de la politique en matière d'armement, basée actuellement sur l'acquisition de la production nationale, pourrait conduire à une évolution vers d'autres formules du fait de l'échec pour l'exportation du Rafale. En effet, le coût des équipements, là aussi souvent difficile à évaluer, grève de façon sensible le budget lorsque les surcoûts se multiplient. Le maintien d'une politique d'armement et d'une industrie national est une question sensible la France restant l'un des principaux producteurs au niveau européen et mondial. La procédure opposée qui consisterait à acheter directement des produits finis au travers d'une mise en concurrence mondiale n'est pas habituelle. Au-delà des surcoûts liés au retard, il ne faut pas négliger aussi ceux liés aux acquisitions en urgence pour répondre à des sous-capacités constatées sur le terrain. Pour conclure sur ce sujet précis, l'industrie de défense n'est impactée que par la commande publique elle-même liée aux ressources affectées pour la politique d'armement. Par conséquent, le fait de réallouer les économies de fonctionnement vers l'investissement ne peut qu'avoir un effet bénéfique pour ce secteur. 


\section{B - Les espaces et leurs enjeux.}

premier lieu, il s'agit des emprises situées en cœur de ville. Traditionnellement, ces terrains ont accueilli les régiments de l'armée de terre. La modernisation des armées et certaines spécialités de l'armée de terre ont rendu difficile le maintient de ces unités dans leurs enceintes originelles pour s'installer en périphérie. Ces bâtiments ont pu toutefois être conservés par l'Etat et accueillent aujourd'hui des services administratifs tels que les centres de recrutement à l'image du Quartier Espagne-La Fayette à Angers, par conséquent les terrains ne sont pas pollués. Même si la qualité des bâtiments est très variable, au regard du développement urbain, la qualité foncière de ces emprises représente une valeur stratégique.

Reste pour les villes et les intercommunalités à cibler dans leur schéma de cohérence territorial l'enjeu posé par la reconversion possible d'un site militaire. La difficulté réside par contre dans l'absence de transcription des espaces militaires en urbanise. Sur un plan, la caserne constitue une tâche blanche là ou chaque parcelle se voit définie selon son usage : habitation, agricole ou commercial, et son état : urbanisé, à urbaniser ou non urbanisable.

Les espaces en périphérie de ville et non pollués constituent la seconde catégorie de cette typologie. Comme évoqué ils ont pu naître du déplacement des unités depuis le cœur de ville ou d'autres villes et confrontés à l'urbanisation existante n'ont pu que s'installer à l'extérieur. Malgré les nuisances potentielles et les mesures de protection liées à ces installations, les sites militaires périphériques sont amenés aujourd'hui à se trouver dans la ville par le jeu de l'étalement urbain. Toutefois, l'enjeu foncier est inférieur au précédent et surtout plus variable. En effet, les contraintes pour limiter l'étalement urbain dans le cadre des lois Grenelle peuvent conduire à restreindre l'intérêt de ces terrains. Ceux situés à proximité de polarités constituent un enjeu de développement à court et moyen terme. Dans certains cas, à l'image de l'Etablissement Technique d'Angers (ETAS) situé à Montreuil-Juigné, il s'agit de terrains spécifiques, des pistes d'essais en l'occurrence. En présence de ces terrains aménagés, une cession à un repreneur pouvant poursuivre l'activité et donc conserver les personnels constitue l'idéal.

23 Enfin, les sites pollués ou trop éloignés sont difficilement valorisables. L'obstacle de la pollution peut-être franchi en contrepartie d'un coût que les acteurs locaux doivent supporter. Lorsque l'engagement d'acquérir est signé, le SID procède aux opérations de dépollution. Selon la nature du site, les opérations peuvent durer de 15 à 30 mois. Afin d'accélérer la procédure, l'acquéreur peut prendre en charge la dépollution pyrotechnique (article 126 de la loi du 4 août 2008 relative à la modernisation de l'économie). Le prix de cessions est net d'un malus tenant compte du coût estimé de dépollution.

L'interrogation qui demeure est celle visant à savoir s'il est préférable de céder ou de conserver l'emprise pour l'Etat. La question vaut essentiellement pour les terrains stratégiques puisqu'il n'y a plus de politique industrielle portée directement par l'Etat mais ces terrains représentent une valorisation économique non négligeable, le but de ces cessions étant de rapporter au budget de l'Etat.

Revue Géographique de l'Est, vol. 51 / 1-2 | 2011 


\section{III - Les acteurs dans la réforme.} immobiliers (DMPA/MRAI) entame le dialogue avec service préfectoraux. Un délégué aux restructurations est assigné à chaque région, il a en charge le suivi de la réforme et des différents projets de reconversion dans sa zone de responsabilité. Les prérogatives du Préfet de région se déclinent vers son homologue département. En effet, le Préfet du département concerné propose la cession du terrain en premier aux communes. Cellesci, en vertu de l'article L 240-1 du code de l'urbanisme sont prioritaires pour l'acquisition des biens de l'Etat. Ce dispositif permet de maintenir le caractère public du bien et qu'il reste dévolu à l'intérêt général. De plus, les communes situées dans le périmètre d'un contrat de redynamisation de site de Défense (CRSD) ont pu demander la cession à l'euro symbolique (le décret $n^{\circ} 2009-829$ du 3 juillet 2009 fixe la liste des communes éligibles au dispositif de cession à l'euro symbolique). Des communes du Calvados, de la Mayenne et de Charente-Maritime sont notamment concernées pour l'aire d'étude. L'accessibilité de ces villes à la liste permet de distinguer les cas critiques dans la réforme.

Les politiques de restructurations confirment le rôle clé que les collectivités sont amenées à jouer à la fois dans l'élaboration du projet de réforme mais surtout dans sa mise en application. Avant la publication de la nouvelle carte militaire, plusieurs collectivités se sont naturellement mobilisées, avec plus ou moins de succès, pour sauvegarder les unités sur leurs territoires. Dans certains cas, l'armée constitue le premier employeur de la commune et des alentours. Au regard de la crise économique qui sévit depuis plusieurs années dans différents secteurs économiques, la sauvegarde réformes, des villes ont déjà pu connaître la disparition de services et donc de fonctionnaires associés comme dans le domaine hospitalier ou judiciaire par exemple. La conjonction de fermeture d'activités est naturellement à éviter et les acteurs se mobilisent pour que le départ d'unités soit compensé.

large communication qu'il réalise sur les restructurations actuelles. La mise en place d'un site internet dédié est une concrétisation. L'utilisateur peut y trouver nombre d'informations sur les objectifs de la réforme ainsi que les mesures mises en œuvre. Par ailleurs, les communications institutionnelles sont fréquentes à l'image d'articles ou de reportages spéciaux dans les périodiques du Ministère. Ainsi, en 2009, un numéro d'Armées d'Aujourd'hui ${ }^{6}$, dressait les perspectives et les enjeux un an après le lancement de la reforme. Nous étions alors encore dans une phase préparatoire puisque quelques Bases de Défenses expérimentales avaient été lancées. Cette propension à communiquer sur le sujet constitue une rupture avec la représentation usuelle véhiculée par l'accolement du terme de "grande muette » aux armées.

effet, plusieurs services internes travaillent sur la restructuration des sites militaires en particulier la direction du mémoire, du patrimoine et des archives (DMPA) et le service des infrastructures de la Défense (SID).

Concrètement, lorsqu'une emprise est libérée, la mission de réalisation des actifs Les services de France Domaine (liés au ministère du Budget) évaluent le bien et fixent le prix de cession. Si la commune décline l'offre, l'emprise peut être cédée à un privé, il y a alors une mise en concurrence pour recueillir les différentes offres. Lorsque le cas 
est complexe, ce qui est fréquent vue la nature des sites militaires, des études peuvent être commandées pour encadrer la reconversion du site. Lorsqu'un privé acquiert une emprise, il peut bénéficier d'aides au travers du Fonds aux Restructurations de la Défense (FRED) pour maintenir l'emploi. Les personnels de la Défense, civils ou militaires, bénéficient de conditions avantageuses s'ils souhaitent quitter l'institution lors de cette réforme et de passerelles pour obtenir un emploi chez un repreneur. Le schéma suivant, réalisé par le ministère de la Défense, recense les différentes étapes de la cession d'une emprise.

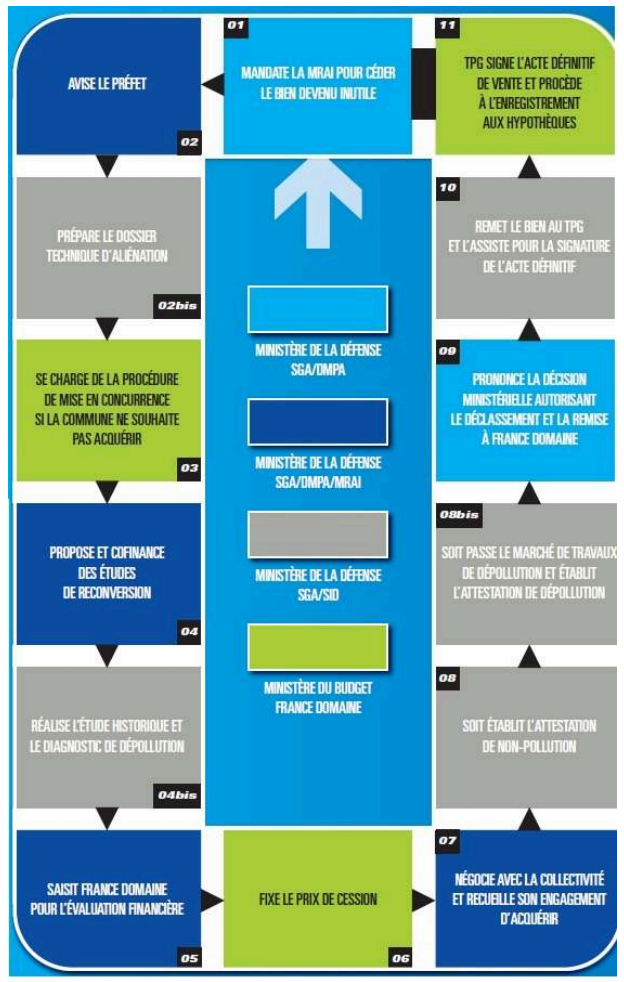

Figure 2 : L'organisation de la cession d'un terrain militaire (source Ministère de la Défense)

Au niveau local, la mobilisation des élus a permis la révision de certaines situations pour compenser ou annuler la fermeture de sites. C'est le cas notamment dans l'Est de la France avec le repositionnement d'une unité de la Brigade Franco-Allemande dans la région. Ce type d'acteur est directement concerné pas les opérations de reconversion puisque les collectivités disposent de la priorité pour l'acquisition des biens de l'Etat. Cette opportunité est particulièrement intéressante pour les villes bénéficiaires de l'acquisition pour l'euro symbolique.

La mise en avant des collectivités ne se fait pas sans difficultés. D'une part, les interrelations avec une multiplicité d'acteurs, parfois en opposition, compliquent les négociations et ajoutent une part d'inertie dans les projets. A titre d'exemple on peut citer le cas de l'aménagement du plateau des Capucins à Brest ${ }^{7}:$ "L'enjeu n'est pas des moindres : faire se rencontrer des mondes professionnels qui s'étaient jusque-là peu fréquentés, d'un côté, la Marine et son ministère de tutelle, et de l'autre, le milieu local brestois des élus et des services de la ville et de la communauté urbaine; bref à l'occasion du désengagement de la Marine sur des sites identifiés, résoudre une tension État/local, soit une recomposition des relations entre l'institution militaire et les 
décideurs urbains vers des accords négociés sur le devenir de la Penfeld. ». Il ne faut pas oublier alors les différences de temporalité entre les agents et les élus.

Si le terrain doit être cédé à un privé, il y a une nécessaire corrélation entre les délégations aux restructurations, une par région, et les agences d'urbanisme et autres syndicats locaux lors de l'étude de dossiers de repreneurs. Ces dossiers peuvent émaner à l'occasion d'un CRSD ou d'un PLR. De manière générale, le Ministère a dressé une fiche signalétique pour chaque emprise dont l'activité peut être reprise par une entreprise.

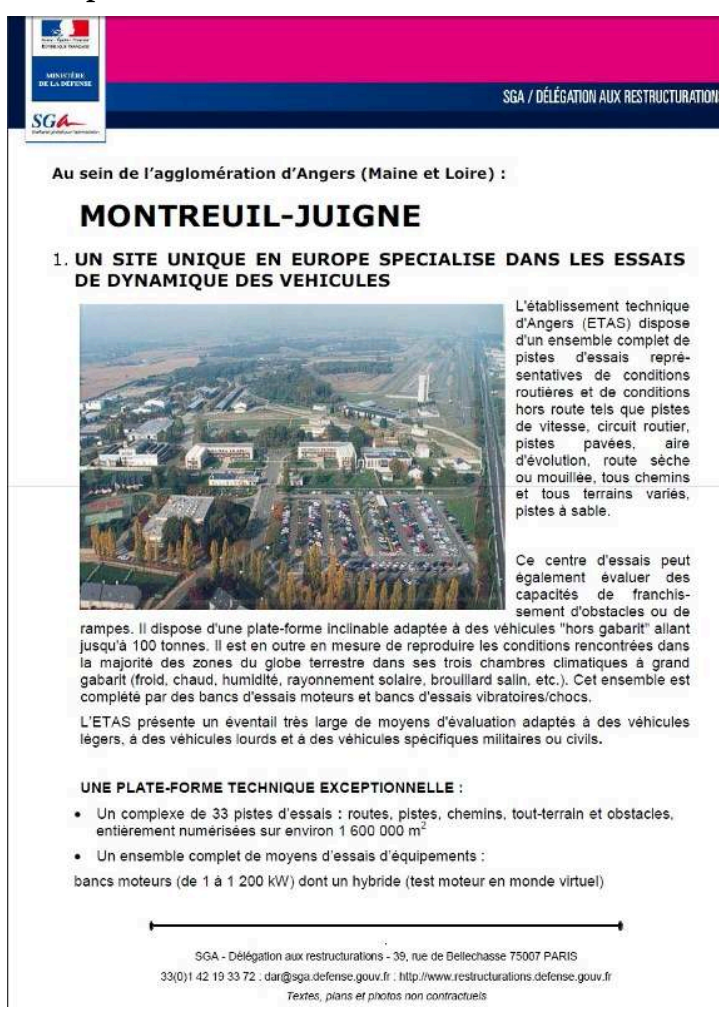

Figure 3 : Extrait de la fiche établie par la Délégation aux Restructurations des Pays-de-la-Loire pour la cession du site de l'Etablissement Technique d'Angers

Les repreneurs privés peuvent obtenir des financements pour l'investissement et le maintien de l'emploi au travers du Fonds pour les Restructurations de la Défense (FRED) doté de $213 \mathrm{M} €$ sur les $320 \mathrm{M} €$ alloués à la réforme (soit environ deux tiers du budget).

Les efforts importants consentis par le Ministère de la Défense et les conditions favorables énoncées en $2008^{8}$ pour l'accompagnement au départ ont conduit à une mobilisation restreinte des personnels civils contre cette réforme. La concertation s'est organisée au niveau des militaires dans les instances représentatives prévues comme le Conseil Supérieur de la Fonction Militaire. Par ailleurs, la nouvelle carte militaire et les grandes étapes de la réforme ont été présentées à l'occasion de sessions plénières réunissant les chefs de corps et commandants de bases.

\section{Conclusion}

Cette réforme d'envergure du Ministère de la Défense en temps de paix conduit à une réadaptation nécessaire de l'organisation territoriale pour mener à bien les différents 
objectifs assignés à la mission de Défense. Les Bases de Défense qui constituent la nouvelle référence au niveau local sont un outil pour parvenir à cet objectif. Les critiques récemment dressées par la Cour des Comptes invitent à poursuivre ce processus de concentration pour parvenir à atteindre la cible budgétaire sans compromettre la capacité des armées à opérer sur différents théâtres comme actuellement. Au-delà des aspects budgétaires cette réforme impacte l'ensemble des acteurs au niveau local. La capacité des collectivités à supporter la fermeture de sites militaires et à développer une nouvelle dynamique à l'occasion des reconversions est primordiale pour réduire le coût social de la réforme. Les effets d'entraînement sur les grands groupes industriels de la Défense dépendent aujourd'hui essentiellement de la Loi de Programmation Militaire, la concentration du secteur et le développement des technologies ayant réduit la sensibilité de la localisation spatiale de ces activités. Toutefois, l'équilibre économique des agglomérations et leurs dynamiques de développement sont dépendante des activités et des échanges qui s'y nouent. En ce sens, le départ d'unités dans des petites et moyennes villes crée un préjudice non négligeable auquel les Plans Locaux de Revitalisation et les Contrats de Redynamisation des Sites de Défense doivent répondre. Dans les agglomérations plus importantes comme Nantes, l'évacuation de sites stratégiques, parce que situés en ville, offre une chance supplémentaire de développement.

\section{BIBLIOGRAPHIE}

Armées d'Aujourd'hui, 2009, Modernisation : l'an 1 de la réforme, Ministère de la Défense $n^{\circ} 343$, p 16-35

BARTHEIL P-A., 2007. Renouvellement urbain à Brest : les enseignements du marché de définition du plateau des Capucins, Norois n' 203, p 7-25

CARRÉ C, 2001, Histoire du Ministère de la Défense, Edition Lavauzelle, 582p

Collectif, 2008, Livre Blanc sur la Défense et la Sécurité Nationale, Editions Odile Jacob, 350p

Collectif (Institut des Hautes Etudes de Défense Nationale), 2006, Comprendre la Défense, Editions Economica, 335p

VOLLET D. et HADJAB F., 2008, Manuel de l'évaluation des politiques publiques, Editions Quae, $64 \mathrm{p}$

\section{Ressources numériques :}

Site Internet de la Révision Générale des Politiques Publiques pour la mission Défense : http:// www.rgpp.modernisation.gouv.fr/index.php?id=36

Site Internet du Ministère de la Défense dédié aux restructurations : http://

www.restructurations.defense.gouv.fr/ 


\section{NOTES}

1. Cette organisation de la pensée est présente dès la table des matières du Livre Blanc dans la troisième partie intitulée: "une nouvelle stratégie pour l'action ", avec les chapitres 9 à 12 dédiés aux quatre piliers issus du Livre Blanc précédent et l'ajout de l'anticipation et la connaissance au chapitre 8.

2. CARRÉ C, 2001, Histoire du Ministère de la Défense, Edition Lavauzelle, 582p

3. Blog de l'Union Nationale des Association de l'Aviation Légère de l'Armée de Terre (UNALAT) http://unaalat.fr/Cour_d_compt_base_def.htm

4. Site du Ministère de la Défense dédié aux restructurations: http:// www.restructurations.defense.gouv.fr/

5. Cette surface a été calculée à partir de la liste des emprises à céder, disponible sur le site Internet dédié aux restructurations (http://www.restructurations.defense.gouv.fr/spip.php? article351)

6. Armées d'Aujourd'hui, 2009, Modernisation : l'an 1 de la réforme, Ministère de la Défense $\mathrm{n}^{\circ} 343, \mathrm{p}$ 16-35

7. BARTHEIL P-A, 2007. Renouvellement urbain à Brest: les enseignements du marché de définition du plateau des Capucins, Norois $\mathrm{n}^{\circ} 203, \mathrm{p} 10$

8. Note du Cabinet du Ministre de la Défense NMR/10652/DEF/CAB du 22 juillet 2008

\section{RÉSUMÉS}

La France de par son histoire entretient un lien étroit avec ses armées. Nombre de villes conservent encore les constructions militaires centenaires, les casernes ayant laissée place parfois à d'autres activités. L'évolution de l'organisation territoriale de la Défense est impactée directement par les modifications physiques du territoire entre expansion et réduction. Cette organisation constitue aussi une adaptation aux choix stratégiques effectués. A partir de 2007, la Révision Générale des Politiques Publiques (RGPP) et le nouveau Livre Blanc de la Défense ont enclenché le mécanisme de la réforme du Ministère de la Défense. En conséquence, une nouvelle disposition des unités sur le territoire a été publiée. La mise en œuvre de la carte militaire regroupant désormais les Bases de Défense implique la fermeture, le déplacement et la création de sites militaires. L'objectif final d'une meilleure efficience de l'outil de Défense crée des bouleversements importants dans les villes concernées. Il apparaît clairement qu'une typologie binaire entre espaces gagnants et perdants est insuffisante. La mobilisation des différentes administrations du niveau national (Ministère) au local (Préfet) est indispensable pour résorber les chocs de ces dynamiques et pour offrir de nouvelles perspectives dans les territoires. La qualité des sites militaires à reconvertir diffère de par leur positionnement géographique mais aussi par l'usage, polluant ou non. Cette dimension impacte lourdement les stratégies à mener pour développer de nouvelles activités. En revanche, au niveau industriel, la corrélation entre la fermeture de site militaire et le déclin industriel d'un territoire n'est plus probant, l'effet sans doute de la concentration dans ce secteur et de l'amélioration des transports.

According to history, France is strongly linked to its armies. Many towns are still keeping military buildings even if there are no more soldiers in it. The evolution of Defence's territorial organization is directly linked to physical land transformation meaning growth or downsizing. 
Since 2007, a public policies benchmark called Revision Générale des Politiques Publiques (RGPP) and the new military doctrine (Livre Blanc de la Défense) have been leading to a wide reform in this department. Consequently, a new military map has been published including closure, translation and opening for some military sites. Defense is to reach, in a more efficiently way, its targets. Regarding troubles caused by this reform, it seems necessary to exceed a binary vision of the issue for towns. The way administrations are acting is a key factor of success to manage economic quakes and to propose a new deal. Military sites are not equal in terms of localization, utility and pollution level that means an adaptation to each case. On the other hand, the link between military desertification and industrial decline is not so strong because of economic concentration et communication improvements.

Frankreich unterhält aus seiner Geschichte heraus eine enge Verbindung zu seinen Armeen. Zahlreiche Städte erhalten die Jahrhunderte alten militärischen Bauten, Kasernen, die manchmal für andere Zwecke frei gestellt wurden. Die Entwicklung der territorialen Organisation der Verteidigung ist unmittelbar von den physikalischen Veränderungen des Territoriums zwischen Expansion und Reduktion beeinflusst. Diese Organisation nahm auch eine Adaptation an die bisherigen strategischen Auswahlmöglichkeiten vor. Von 2007 an haben die Révision Générale des Politiques Publiques (RGPP) und das neue Weißbuch zur Verteidigung den Anstoß zur Reform des Verteidigungsministeriums gegeben. Infolgedessen wurde eine neue Verordnung über die territorialen Einheiten veröffentlicht. Die Anfertigung der militärischen Landkarten bezieht die Schließung, die Auslagerung und Neuerrichtung von militärischen Anlagen mit ein. Das angestrebte Ziel einer besseren Effizienz der Verteidigung schafft wichtige Umwälzungen in den davon betroffenen Städten. Es scheint offensichtlich zu sein, dass eine binäre Typologie, die zwischen Raumgewinn und Raumverlust unterscheidet, unzureichend ist. Die Mobilisierung der unterschiedlichen Verwaltungen auf nationalem (Ministerium) und lokalen Niveau (Präfektur) ist unerlässlich, um den Schock aus dieser Dynamik abzumildern und neue Perspektiven für die Territorien anzubieten. Die Qualität der militärischen Anlagen, die umgewandelt werden sollen, unterscheidet sich durch die geographische Lage, aber auch durch ihre Umwelt schonende oder nicht Umwelt schonende Ausführung. Diese Dimension erfordert in besonderer Weise Strategien, um neue Aktivitäten zu entwickeln. Dagegen hat auf industriellem Niveau die Korrelation zwischen der Schließung einer militärischen Anlage und dem industriellen Niedergang eines Gebietes keine größere Aussagekraft, das ist ohne Zweifel eine Folge der Konzentration in diesem Sektor und der Verbesserung der Transport- und Kommunikationswege.

INDEX

Mots-clés : défense, carte militaire, Base de Défense, enjeux territoriaux

Keywords : Defense, military map, military basis, territorial stakes

Schlüsselwörter : Verteidigung, militärische Landkarte, Verteidigungsbasis, territoriale Probleme

\section{AUTEUR}

FLORIAN RAPIN

Doctorant en géographieUniversité d'Angers / Laboratoire Espaces et Sociétés 